K. MAEHARA

KODAI MATH. J.

11 (1988), $1-4$

\title{
THE MORDELL-BOMBIERI-NOGUCHI CONJECTURE OVER FUNCTION FIELDS
}

\author{
BY KAZUHISA MaEHARA
}

§1. Introduction. G. Faltings recently proved the Mordell conjecture [F]. The author learned from J. Noguchi that E. Bombieri made the following conjecture generalizing the conjecture above (cf. also [L]):

The set of rational points of any projective variety of general type over an algebraic number field is not Zariski dense.

Noguchi ([N1], [N2]) has obtained some results over function fields which are analogues of the Bombieri conjecture.

CONJECTURE A (Noguchi). Let $f: X \rightarrow S$ be a proper surjective map between non-singular projective varieties over the complex number field. Let $\sigma_{\lambda}$ denote the rational sections of $f$. Assume that a general fibre $X_{s}$ of $f$ is a variety of general type and that the union of $S_{\lambda}=\sigma_{\lambda}(S)$ is Zariski dense in $X$. Then $X$ is birationally trivial, i.e., there exists a projective variety $X_{0}$ such that $X$ is birational to $X_{0} \times S$.

We pose the following conjecture, which implies Conjecture A.

CONJECTURE B. Let $X$ and $S$ be non-singular projective varieties. Then there exists an ample divisor $D$ on $S$ such that for any birational embedding $j_{\lambda}$ : $S \rightarrow X$ we have $\mathcal{O}\left(j_{\lambda}^{*} K_{X}\right) \subset \mathcal{O}(D)$.

Note that when $X$ is the minimal model of a surface with $\kappa(X) \geqq 0$, Miyaoka and Umezu proved Conjecture $\mathrm{B}([\mathrm{MU}])$. We shall prove Conjecture A with additional assumptions:

MAIN THEOREM. Let $f: X \rightarrow S$ be a proper surjective map between nonsingular projective varieties over the complex number field. Let $\sigma_{\lambda}$ denote the rational sections of $f$. Assume that a general fibre $X_{s}$ of $f$ is a variety of general type and the union of $S_{\lambda}=\sigma_{\lambda}(S)$ is Zariski dense in $X$. Let $P$ denote the projective bundle $p: P\left(\Omega_{X}^{s}\right) \rightarrow X$, where $s=\operatorname{dim} S$. Furthermore suppose that $O(\alpha)$ $\otimes p^{*} \mathcal{O}\left(-K_{X}\right)$ is $f \circ p-n e f$ for some $\alpha>0$. Then $X$ is birationally trivial over $S$.

Received May 7, 1987 


\section{$\S 2$. Proof of Main Theorem.}

We reduce the proof of Main Theorem to the next lemma ([M1], section 5(p. 115), Appendix (p. 119)), which is derived from the weak positivity of direct image sheaf of a high multiple of the relative dualizing sheaf ([KMM], [Ko1], [M2], [V]).

LEMMA 1. Let $T$ be a complete variety and $\phi: T \times S \rightarrow X$ be a dominant $S$ rational map. Then $X$ is birationally trivial over $S$.

For this purpose it suffices to show that there exists a dense bounded subfamily of the graphs of rational sections.

LEMmA 2. We let $S_{\lambda}=\sigma_{\lambda}(S)$ and $S_{\lambda}^{o}$ denote the regular part of $S_{\lambda}$. The natural surjection $\left.\Omega_{X}^{s}\right|_{S_{\lambda}^{o}} \rightarrow \omega_{S_{\lambda}^{o}}^{o}$ gives a unique $X$-map $S_{\lambda}^{o} \rightarrow P$.

Proof. The natural surjection $\left.\Omega_{X}\right|_{S_{\lambda}} \rightarrow \Omega_{S_{\lambda}}$ induces a surjection $\left.\Omega_{X}^{s}\right|_{s_{\lambda}^{o} \rightarrow} \rightarrow$ $\Omega_{S_{\lambda}^{o}}^{s_{0}}=\omega_{S_{\lambda}^{o}}^{o}$. Hence from universality of $P$, we have the unique $X$-map $s_{\lambda}: S_{\lambda}^{o} \rightarrow P$ such that the pull-back by $s_{\lambda}$ of the surjection $p^{*} \Omega_{X}^{s} \rightarrow \mathcal{O}(1)$ coincides with $\Omega_{X}^{s} \mid s_{\lambda}^{o}$ $\rightarrow \omega_{S_{\lambda}^{o}}^{o}$. Q.E.D.

We denote by $G_{\lambda}$ the graph in $S \times P$ of the composition of the rational sections $s_{\lambda}: S_{\lambda} \rightarrow P$ and $\sigma_{\lambda}: S \rightarrow S_{\lambda}$. Let $B$ and $H$ be suitable ample invertible sheaves on $S$ and $X$, respectively. We put

$$
M=\operatorname{pr}_{1}^{*} B \otimes \operatorname{pr}_{2}^{*}\left(\left(\mathcal{O}(\alpha) \otimes p^{*} \omega_{X}^{-1}\right)^{k} \otimes p^{*} H\right)^{\beta},
$$

where the $\operatorname{pr}_{\imath=1,2}$ are the projections from $S \times P$ onto $S$ and $P$, respectively. We estimate the Hilbert polynomials of subvarieties $\left\{G_{\lambda}\right\}$ of $S \times P$ with respect to $M$.

LEMmA 3. For a dense subset $\left\{G_{\lambda}\right\}, \chi\left(G_{\lambda}, M^{m}\right)$ are a finite number of polynomials in $\mathrm{m}$.

Proof. From our assumption, $O(\alpha) \otimes p^{*} \omega_{X}^{-1}$ is $f \circ p$-nef for some $\alpha>0$. Since $\mathcal{O}(\alpha) \otimes p^{*} \omega_{X}^{-1}$ is $p$-ample, we can take $H$ such that $\mathcal{O}(\alpha) \otimes p^{*} \omega_{X}^{-1} \otimes p^{*} H$ is ample. Hence for any $k>0,\left(\mathcal{O}(\alpha) \otimes p^{*} \omega_{X}^{-1}\right)^{k-1+1} \otimes p^{*} H$ is $f \circ p$-ample. We choose $k$ such that $\kappa\left(\left(\omega_{X} \otimes f^{*} A\right)^{k} \otimes H^{-1}\right) \geqq 0$, where $A$ is an ample invertible sheaf on $S$. In fact, by the Viehweg formula $\kappa\left(\omega_{X} \otimes f^{*} A\right)=\kappa\left(\omega_{X_{s}}\right)+\operatorname{dim} S$ ([V]), $\omega_{X} \otimes f^{*} A$ is big. We take $\beta>0$ so that $\left(\left(\omega_{X} \otimes f^{*} A\right)^{k} \otimes H^{-1}\right)^{\beta}$ is effective. Let $\mu_{\lambda}: F_{\lambda} \rightarrow G_{\lambda}$ be a desingularization of $G_{\lambda}$ such that $a_{\lambda}: F_{\lambda} \rightarrow S_{\lambda}$ and $\nu_{\lambda}: F_{\lambda} \rightarrow S$ are at the same time resolutions of a birational map $G_{\lambda} \rightarrow S_{\lambda}$, i. e., the inverse of the composition of $\bar{S}_{\lambda}=s_{\lambda}\left(S_{\lambda}\right) \rightarrow S_{\lambda}$ and $\mathrm{pr}_{2}: G_{\lambda} \rightarrow \bar{S}_{\lambda}$ and a birational map $G_{\lambda} \rightarrow S$, i. e., the inverse of the composition of $S_{\lambda} \rightarrow S, \bar{S}_{\lambda} \rightarrow S_{\lambda}$ and $\mathrm{pr}_{2}: G_{\lambda} \rightarrow \bar{S}_{\lambda}$.

We have the following diagram: 


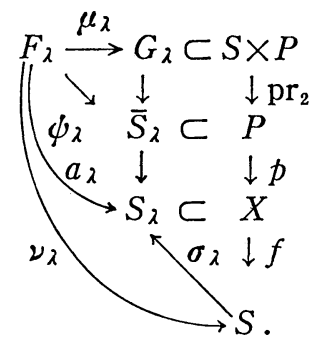

Here we denote by $\phi_{\lambda}$ the composition of the projection $\left.\operatorname{pr}_{2}\right|_{G_{\lambda}}: G_{\lambda} \rightarrow \bar{S}_{\lambda}$ and $\mu_{\lambda}: F_{\lambda} \rightarrow G_{\lambda}$. We have the natural homomorphism $\theta_{\lambda}: a_{\lambda}^{*} \Omega_{X}^{s} \rightarrow \Omega_{F_{\lambda}}^{s}=\omega_{F_{\lambda}}$ induced from $a_{\lambda}^{*} \Omega_{X} \rightarrow \Omega_{F_{\lambda}}$. Then the image $\operatorname{im} \theta_{\lambda}$ of $\theta_{\lambda}$ is torsion free. Therefore there exists an open immersion $F_{\lambda}^{o} \subset F_{\lambda}$ with $\operatorname{codim}\left(F_{\lambda} \backslash F_{\lambda}^{o}\right) \geqq 2$ such that $\operatorname{im} \theta_{\lambda}$ is invertible over $F_{\lambda}^{o}$. By Lemma $2, \theta_{\lambda}$ is nothing but $\phi_{\lambda}^{*} p^{*} \Omega_{X}^{s} \rightarrow \psi_{\lambda}^{*} \mathcal{O}(1)$ over $F_{\lambda}^{o}$. Hence the double dual $(\operatorname{im} \theta) \approx$ coincides with $\psi_{\lambda}^{*} \mathcal{O}(1)$. Moreover we have

$$
\left(\operatorname{im} \theta_{\lambda}\right) \check{\Sigma}=i_{*}\left(\left.\operatorname{im} \theta_{\lambda}\right|_{F_{\lambda}^{o}}\right) \longrightarrow i_{*}\left(\omega_{F_{\lambda}^{o}}\right)=\omega_{F_{\lambda}} .
$$

Thus $\operatorname{1m} \theta_{\lambda}=\phi_{\lambda}^{*} \Theta(1)$. We obtain

$$
\text { (1) } \phi_{\lambda}^{*} O(1) \subset \omega_{F_{\lambda}} \text {. }
$$

We shall estimate the following polynomials $\chi\left(G_{\lambda}, \mu_{\lambda *} \omega_{F_{\lambda}} \otimes M^{m}\right)$ in $m$, which equals $\operatorname{dim} H^{0}\left(F_{\lambda}, \omega_{F_{\lambda}} \otimes M^{m}\right)$ for $m \geqq 1$ by the Kollár vanishing ([Ko1]). We have for a dense subset $\left\{G_{\lambda}\right\}$

$$
\begin{aligned}
& \operatorname{dim} H^{0}\left(F_{\lambda}, \omega_{F_{\lambda}} \otimes M^{m}\right)=h^{0}\left(F_{\lambda}, \omega_{F_{\lambda}} \otimes \nu_{\lambda}^{*} B^{m} \otimes \psi_{\lambda}^{*}\left(\left(O(\alpha) \otimes p^{*} \omega_{X}^{-1}\right)^{k} \otimes p^{*} H\right)^{\beta m}\right) \\
& \leqq h^{0}\left(F_{\lambda}, \omega_{F_{\lambda}} \otimes \nu_{\lambda}^{*} B^{m} \otimes \omega_{F_{\lambda} \beta}^{k m} \otimes p^{*} f^{*} A^{k m \beta}\right) .
\end{aligned}
$$

By the projection formula, we get

$$
\begin{aligned}
& h^{0}\left(F_{\lambda}, \omega_{F_{\lambda}} \otimes \nu_{\lambda}^{*} B^{m} \otimes \omega_{F_{\lambda}}^{k m \beta} \otimes p^{*} f^{*} A^{k m \beta}\right) \\
& =h^{0}\left(F_{\lambda}, \omega_{F_{\lambda}}^{1+k m \beta} \otimes \nu_{\lambda}^{*}\left(B^{m} \otimes A^{k m \beta}\right)\right) \\
& =h^{0}\left(S, \omega_{S}^{1+k m \beta} \otimes B^{m} \otimes A^{k m \beta}\right) \quad \text { for } \quad m \geqq 1 .
\end{aligned}
$$

Therefore there exist only a finite number of polynomials in $m$ in the form $\chi\left(G_{\lambda}, \mu_{\lambda_{*} \omega_{F}} \otimes M^{m}\right)$. From Kleiman ([KL]), $\chi\left(G_{\lambda}, M^{m}\right)$ are also a finite number of polynomials in $m$. Q.E.D.

We now return to the proof of Main Theorem. The next lemma implies Main Theorem since its assumption is already shown in Lemma 3.

LEMMA 4. Let $\left\{G_{\lambda}\right\} \subset S \times P$ be a set of graphs of rational sections from $S$ to $P$ with a finite number of Hilbert polynomial $\chi\left(G_{\lambda}, M^{m}\right)$. Suppose that there exist rational sections corresponding to $\left\{G_{\lambda}\right\}$ which form a Zariski dense subset 


\section{in $X$. Then $X$ is birationally trivial over $S$.}

Proof. By the same argument as in [M1], we find an open subset $T^{\circ}$ of the Hilbert scheme with a Hilbert polynomial such that all the points of $T^{\circ}$ correspond to the graphs of rational sections from $S$ into $P$. From our assumption that there exist only a finite number of Hilbert polynomials, we find $T^{0}$ such that $T^{o}$ parametrizes a dense set of rational sections in $X$. Let $G$ denote the universal family restricted on $T^{\circ}$. Then we obtain a $T^{\circ}$-birational morphism $G \rightarrow S \times T^{\circ}$ and a $T^{\circ}$-morphism $G \rightarrow P \times T^{o}$, which induces a $T^{\circ}$-map $S \times T^{o} \rightarrow P \times T^{\circ}$. Since $T^{0}$ contains points associated to rational sections $\left\{\sigma_{\lambda}\right\}$ forming a Zariski dense subset, we obtain a dominant $S$-map $F^{o}: S \times T^{\circ} \rightarrow X$, composing $S$-maps $S \times T^{o} \rightarrow P \times T^{o}$ and $P \times T^{o} \rightarrow P \rightarrow X$. Taking a smooth compactification $T$ of $T^{o}$, we have a dominant $S$-rational map $S \times T \rightarrow X$. Thus Lemma 1 implies that $X$ is birationally trivial over S. Q.E.D.

\section{REFERENCES}

[F] G. Faltings, Arakelov's theorem for Abelian varieties, Invent. Math., 73 (1983), 337-348.

[KMM] Y. Kawamata, K. Matsuki and M. Matsuda, Introduction to the minimal model problem, Advanced Studies in Pure Math., 10, Algebraic Geometry, pp. 283-360, Sendai (1985), 1987.

[KL] S. Kleiman, Les theoremes de finitude pour le foncteur de Picard, Fx. X, SGA 6, Lecture Notes in Math., 225, Springer, Berlin-Heiderberg-New York, 1971.

[Ko1] J. KolláR, Higher direct images of dualizing sheaves I, Ann. Math., 123 (1986), 11-42.

[Ko2] J. KolláR, Higher direct images of dualizing sheaves II, Ann. Math., 124 (1986), 171-202.

[L] S. LANG, Hyperbolic and diophantine analysis, Bull. Amer. Math. Soc., 14 (1986), 159-205.

[M1] K. MAeHARA, A finiteness property of varieties of general type, Math. Ann. 262 (1983), 101-123.

[M2] K. Maehara, The weak 1-positivity of direct image sheaves, J. reine und angewante Math., 364 (1986), 112-129.

[MU] Y. MiYaoka AND Y. UmezU, The boundedness of curves with fixed genus on minimal surfaces, manuscript.

[N1] J. NoGUCHI, A higher dimensional analogue of Mordell's conjecture over function fields, Math. Ann., 258 (1981), 207-212.

[N2] J. NoGUCHI, Hyperbolic fibre spaces and Mordell's conjecture over function fields, Publ. RIMS, Kyoto Univ., 21 (1985), 27-46.

[V] E. VIEHWEG, Weak positivity and the additivity of the Kodaira dimension for certain fiber spaces, in Algebraic Varieties and Analitic varieties, Advanced Studies in Pure Math., 1, pp. 329-353, Tokyo and Amsterdam, 1983.

Tokyo Institute of Polytechnics

1583 IIYAma, Atsugi

KANAGAWA, 243-02 JAPAN 